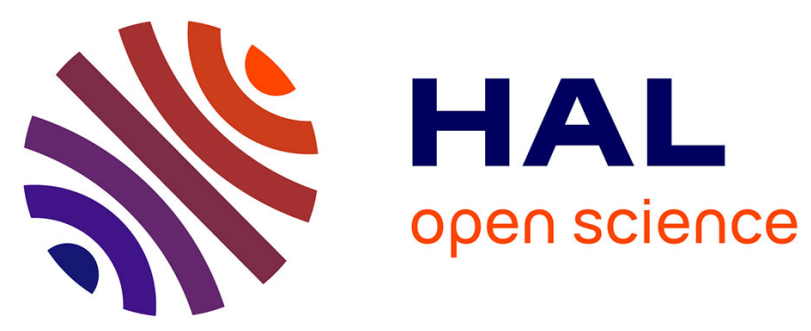

\title{
Testbed for assessing the accuracy of interventional radiology simulations
}

Mario Sanz Lopez, Jérémie Dequidt, Erwan Kerrien, Christian Duriez, Marie-Odile Berger, Stéphane Cotin

\section{To cite this version:}

Mario Sanz Lopez, Jérémie Dequidt, Erwan Kerrien, Christian Duriez, Marie-Odile Berger, et al.. Testbed for assessing the accuracy of interventional radiology simulations. ISBMS - 6th International Symposium on Biomedical Simulation, Oct 2014, Strasbourg, France. hal-01059892

\section{HAL Id: hal-01059892 \\ https://inria.hal.science/hal-01059892}

Submitted on 2 Sep 2014

HAL is a multi-disciplinary open access archive for the deposit and dissemination of scientific research documents, whether they are published or not. The documents may come from teaching and research institutions in France or abroad, or from public or private research centers.
L'archive ouverte pluridisciplinaire HAL, est destinée au dépôt et à la diffusion de documents scientifiques de niveau recherche, publiés ou non, émanant des établissements d'enseignement et de recherche français ou étrangers, des laboratoires publics ou privés. 


\title{
Testbed for Assessing the Accuracy of Interventional Radiology Simulations
}

\author{
Mario Sanz-Lopez ${ }^{1}$, Jeremie Dequidt ${ }^{2}$, Erwan Kerrien ${ }^{1,3}$, Christian Duriez ${ }^{1,2}$, \\ Marie-Odile Berger ${ }^{1,3}$ and Stephane Cotin ${ }^{1,2}$ \\ 1 INRIA, France \\ ${ }^{1}$ University of Lille, France \\ ${ }^{3}$ University of Nancy, France
}

\begin{abstract}
The design of virtual reality simulators, and more specifically those dedicated to surgery training, implies to take into account numerous constraints so that simulators look realistic to trainees and train proper skills for surgical procedures. Among those constraints, the accuracy of the biophysical models remains a very hot topic since parameter estimation and experimental validation often rely on invasive protocols that are obviously not suited for living beings. In the context of Interventional Radiology the procedures involve the navigation of surgical catheter tools inside the vascular network where many contacts, sliding and friction phenomena occur. The simulation of these procedures require complex interaction models between the tools and the blood vessels for which there is no ground truth data available for parametrization and validation. This paper introduces an experimental testbed to address this issue: acquisition devices as well as a data-processing algorithms are used to record the motion of interventional radiology tools in a silicon phantom representing a vascular network. Accuracy and high acquisition rates are the key features of this testbed as it enables to capture dynamic friction of non-smooth dynamics and because it could provide extensive data to improve the accuracy of the mechanical model of the tools and the interaction model between the tools and the blood vessel.
\end{abstract}

\section{Introduction}

Interventional radiology procedures rely on the navigation of a catheter inside the blood vessels through fluoroscopic image guidance. When the tip of the catheter is close to the location of the disease, dedicated tools (coils, stents, balloon...) can be deployed or drugs can be delivered in order to diagnose or treat the patient. To control the motion of a catheter within the vascular network, the radiologist can only push, pull or twist the proximal end of the device. She/he also can insert a stiffer device, named guidewire, that will help reaching specific locations. Since such devices are constrained inside the patient's vasculature, the motion of the devices results from the displacement inputs of the radiologist as well as contact forces between the devices and the arterial walls. These contact forces are particularly important since the motion of the devices is greatly 
affected by dynamic effects such as friction or sliding which can significantly change the trajectory followed by the devices. Even if the friction between the arterial wall and the tools may be negligible in certain circumstances (for instance slow motion, simple path to reach the targeted area), its effects are significant in tortuous vasculature or aneurysms. The figure 1 illustrates that friction implies high frequency motion for the deployment of a coil (thin coated platinum thread) in an aneurysm. Therefore, accurate models for the tools and for the interactions between tools and the blood vessel are mandatory. However, the quantitative evaluation of the accuracy is hard to perform due to the complexity of the environment: while imaging techniques allow to capture the geometry of the anatomical structures, the bio-mechanical parameters are hard to measure / estimate. Some previous work has proposed strategies to estimate the accuracy of their simulations: Dequidt et. al. [4] use 3D rotational angiography to evaluate the simulation of a coil deployment in a collision-free environment. Luboz et. al. [5] compare the position of simulated guidewires and actual guidewires inserted in a vascular phantom. The comparison is however performed on equilibrium positions and on a almost linear trajectory which reduces the influence of friction. Finally, Alderliesten et. al [1] have conducted a thorough quantitative experimental validation of a quasi-static model of guidewires: 3D comparison through 3DRA is performed with several deployment scenarios. However the main limitation of the approach is that only static friction is considered and the friction parameter is empirically set. Even if the friction between the arterial wall and the tools may be negligible in certain circumstances, its effects are significant in tortuous vasculature or aneurysms even if special care is taken by the radiologist with slow and delicate manipulations. For instance, friction implies high frequency motion for the deployment of a coil (thin coated platinum thread) in an aneurysm. To capture this effect on navigation scenarios, our objective is to design a testbed with software support that is able to record dynamic and non-smooth phenomena (i.e high speed changes). This paper describes an experimental testbed suited for navigation scenarios that include motion tracking of the tool but also image tracking through high-speed stereoscopic cameras.

\section{Validation testbed}

The testbed proposed in this paper is designed to provide ground-truth data to compare against simulation. To do so, the testbed is composed of two components: (1) a tracking device is built to accurately track the input motion of the surgeon navigating the radiology tools, (2) high-speed stereoscopic cameras coupled with segmentation and reconstruction provide the position of the tool in a vascular silicon phantom.

Motion Tracking: We have designed a new motion tracking interface, based on an optical sensor from a mouse that detects motion with a 1200 dpi resolution, a standard HID USB interface and a radio wireless connexion to transmit the raw data without cluttering the operating field. A new package has been built 

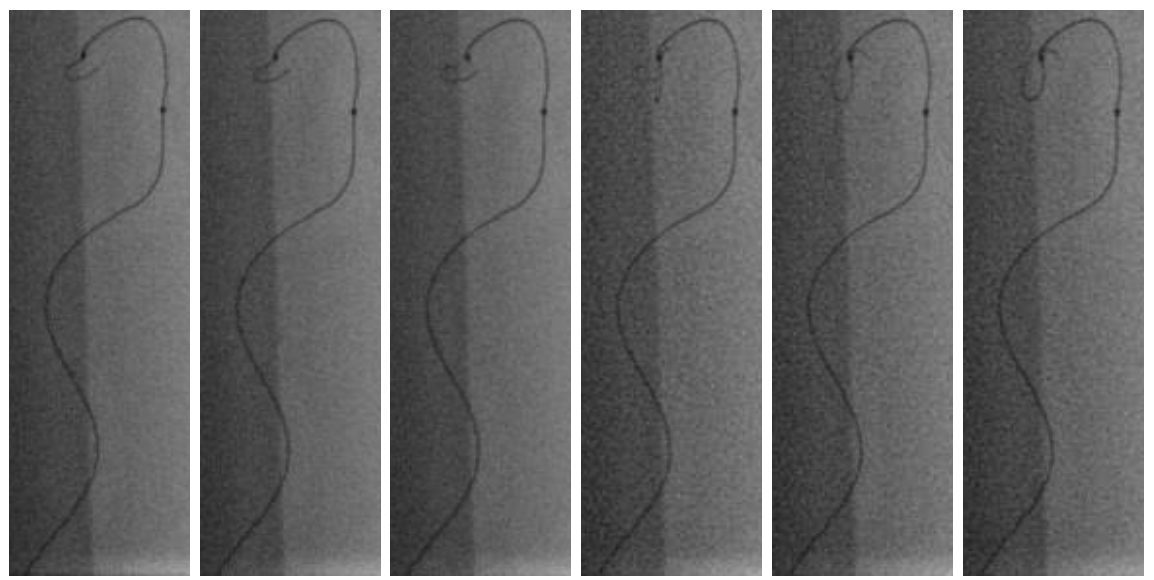

Fig. 1: Recorded sequence of a coil deployed at low and constant speed where image acquisition is performed at 15 fps through 3D Rotational Angiography. The sequence shows the effect of friction and then sliding as the coil completely changes its configuration between frames 3 and 4 ( 67 msecs between each frame).

for the sensor allowing an easy handle, standard surgical attach systems on both sides and removable tunnels adapted to each size of catheters and coils. The main issues with the use of a mouse sensor relies in sensing the cylinder shape of the catheter instead of a flat surface, the placement of the circuit in a different embodiment, as well as the existence of a wireless, potentially noisy link rise questions about its reliability. Moreover, the data provided is differential, which could lead to a cumulative input error. Thus, a precision test has been conducted considering both local (resolution) and absolute accuracy using a calibrated test bench.

This test bench is composed of a motorized spool that pulls over one meter of bare electric wire, with a negligible elongation factor and a constant diameter of $1.54 \mathrm{~mm}$ (close to interventional radiology tools). While the electric wire diameter
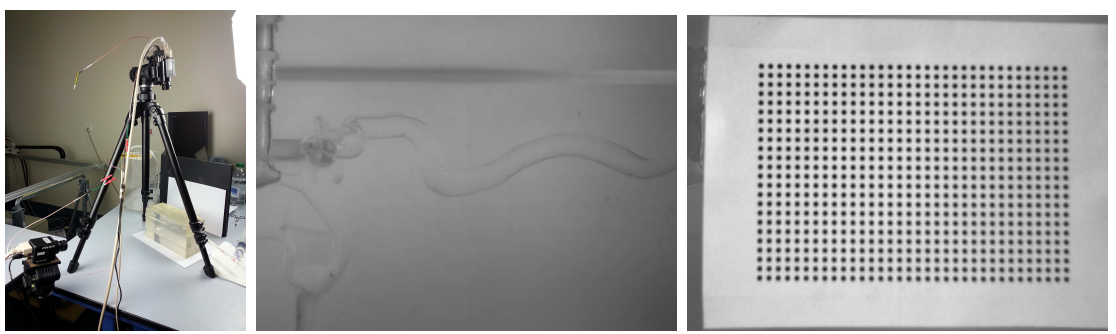

Fig. 2: (left) Experimental setup, showing the top and side camera, observing the silicon vascular phantom; (middle) image of the phantom by the top camera; (right) calibration pattern used to compute the equation of the refractive plane (inter-bullet distance $=2 \mathrm{~mm}$ ) 

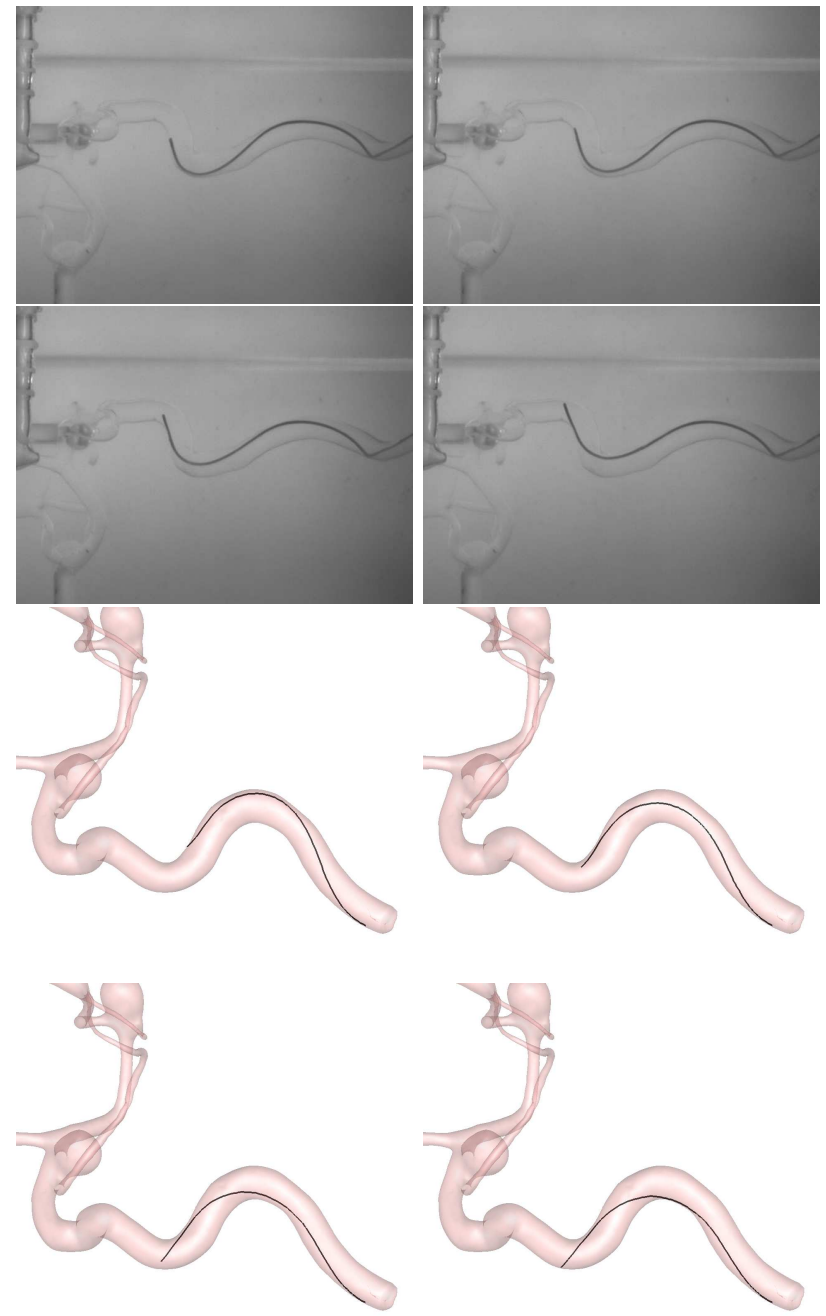

Fig. 3: Stick and slip transition: (top) top camera raw view, (bottom) reconstruction. Only one out of two images of the original sequence were shown for a better readability. A top speed of $485.13 \mathrm{~mm} / \mathrm{s}$ was recorded at the start of the sequence. 
is greater that the actual interventional radiology tools, it was chosen due to its non-elongation property and we use dedicated diameter-dependent removable tunnels that allow to place any thin wire in the focal plane of the sensor in order to maximize the motion tracking. Moreover the wire is not insulated as it does not provide a significant difference for the tracking. The motion of the spool is tracked using the encoder of a ball mouse and controlled through a microcontroller connected to power interfaces driving two motors at both sides of the cylinder; the grip between the encoder wheel and the spool is ensured by choosing a silicone rubber solid cylinder for the spool, with typical friction coefficient larger than 1 . Under these conditions, the mouse sent exactly 1260 counts, which gave a final system absolute error of $\pm 396 \mu m$, that can be considered negligible. A landmark was set on the wire in order to compare after each test that the mark was perfectly aligned with the goal position of 1 meter of wire pulled.

The laser mouse used as sensor makes data easy to receive, but it needs to be fetched before the Operating System applies transfer functions and denaturalizes the raw recovered data, this was done with the libpointing library [2]. 6 tests were conducted at various speeds and the distance recorded by the sensor was stored. For 1 meter of wire pulled with a speed of $(0.0487,0.0967$, $0.145,0.192,0.242$ and $0.290 \mathrm{~m} / \mathrm{s})$ the sensed distance was $(1051.200,1006.284$, $1009.480,1064.662,1060.026$ and $991.4255 \mathrm{~mm}$ ) which leads to an average error of $3.3 \%$.

High-speed Stereoscopic Tracking: The system was centered on two JAI-Pulnix TM-6740CL cameras configured to acquire $640 \times 480$ grayscale images at 198 frames per second. Synchronization was performed thanks to a trigger (CC320 Machine Vision Trigger Timing Controller, Gardasoft). Two Super Cool-Lite 9 (interFit Lighting) projectors were used to reduce the flickering observed with neon lights. Both cameras were placed to respectively look down and sideways (see Fig. 2, left). The stereo pair of cameras were calibrated using chessboard detection and calibration by OpenCV.

The vasculature was given by a phantom made of silicon (right internal carotid artery rigid model with three aneurysms, by Elastrat). Silicon is transparent (see Fig. 2, middle) but induces refraction that significantly perturbs the epipolar geometry of the stereoscopic cameras and forbids using traditional 3D reconstruction based on the triangulation of visual rays [3]. The plane equations of both refractive faces were given by computing the associated homography thanks to a calibration pattern and OpenCV (see Fig. 2, right).

We used a straight shaped microcatheter with an outside diameter of 1.7 French (Headway ${ }^{\mathrm{TM}} 17$ regular Microcatheter, Microvention) that was only pushed into the phantom. The microcatheter could automatically be segmented after subtracting a catheter-free image. Refractive stereoscopic reconstruction was thereafter applied followed by an RBF (Radial Basis Functions) smoothing of the curve. The reconstruction error was estimated to be below $0.05 \mathrm{~mm}$ on 1000 images of a motionless catheter. A 3D model of the phantom vasculature was segmented from 3 DRA data using $[6,7]$ and manually registered with the images of both cameras. In order to estimate the imprecision of the reconstruction, 
we measured the average displacement of the catheter tip at the start of the sequence, while no motion was applied (1000 images): a standard deviation of $[0.0075,0.01750 .0457] \mathrm{mm}$ was observed along the $\mathrm{X}-\mathrm{Y}-\mathrm{Z}$ axes ( $\mathrm{Y}$ and $\mathrm{Z}$ axes are respectively along the optical axes of the side and top cameras).

\section{Results and Conclusion}

We focused our analysis on abrupt motions. Fig. 3 gives an example of a stick and slip transitions: starting from a static position of the catheter tip, a motion amplitude of $10.1 \mathrm{~mm}$ was observed on 8 consecutive images, which gives an average speed of $286.86 \mathrm{~mm} / \mathrm{s}$. Over this sequence, the instantaneous speeds, as measured in-between two consecutives images, were : [7.06, 123.74, 485.13, $400.04,388.54,388.98,326.56,27.75] \mathrm{mm} / \mathrm{s}$. A peek of almost $500 \mathrm{~mm} / \mathrm{s}$ was observed at the start of the sequence.

These first results demonstrate that we have developed a testbed able to validate numerical models and simulations of the mechanical interaction between catheter and blood vessels. In future work, we will use this experimental ground truth to validate and compare existing simulation algorithms. The testbed has been built using a rigid silicon phantom as it allows to simplify the actual environment and puts the emphasis on the tool model and the interaction model between the tool and the blood vessel. Using a soft silicon phantom will be considered in a future work to validate vessel-deformation models but will require a more complex machinery (regular segmentations of the vasculature will be mandatory to capture the deformations of the soft silicon phantom).

\section{References}

1. Alderliesten, T., Konings, M., Niessen, W.: Modeling friction, intrinsic curvature, and rotation of guide wires for simulation of minimally invasive vascular interventions. Biomedical Engineering, IEEE Transactions on 54(1), 29-38 (Jan 2007)

2. Casiez, G., Roussel, N.: No more bricolage!: Methods and tools to characterize, replicate and compare pointing transfer functions. In: UIST. pp. 603-614 (2011)

3. Chari, V., Sturm, P.: Multiple-view geometry of the refractive plane. In: BMVC 2009 - 20th British Machine Vision Conference. pp. 1-11 (Sep 2009)

4. Dequidt, J., Marchal, M., Duriez, C., Kerien, E., Cotin, S.: Interactive simulation of embolization coils: Modeling and experimental validation. In: MICCAI. pp. 695-702 (Sep 2008)

5. Luboz, V., Zhai, J., Odetoyinbo, T., Littler, P., Gould, D., How, T., Bello, F.: Simulation of endovascular guidewire behaviour and experimental validation. Computer methods in biomechanics and biomedical engineering 14(06), 515-520 (2011)

6. Yureidini, A., Kerrien, E., Cotin, S.: Robust RANSAC-based blood vessel segmentation. In: Haynor, D.R., Ourselin, S. (eds.) SPIE Medical Imaging. vol. 8314, p. 8314M. SPIE Press (Feb 2012)

7. Yureidini, A., Kerrien, E., Dequidt, J., Duriez, C., Cotin, S.: Local implicit modeling of blood vessels for interactive simulation. In: Ayache, N., Delingette, H., Golland, P., Moria, K. (eds.) MICCAI. Lecture Notes in Computer Science (Oct 2012) 